\title{
Efficient Calculation of Born Scattering for Fixed-Offset Ground-Penetrating Radar Surveys
}

\author{
Meincke, Peter
}

Published in:

I E E E Geoscience and Remote Sensing Letters

Link to article, DOI:

10.1109/LGRS.2006.886423

Publication date:

2007

Document Version

Publisher's PDF, also known as Version of record

Link back to DTU Orbit

Citation (APA):

Meincke, P. (2007). Efficient Calculation of Born Scattering for Fixed-Offset Ground-Penetrating Radar Surveys. I E E E Geoscience and Remote Sensing Letters, 4(1), 88-92. https://doi.org/10.1109/LGRS.2006.886423

\section{General rights}

Copyright and moral rights for the publications made accessible in the public portal are retained by the authors and/or other copyright owners and it is a condition of accessing publications that users recognise and abide by the legal requirements associated with these rights.

- Users may download and print one copy of any publication from the public portal for the purpose of private study or research.

- You may not further distribute the material or use it for any profit-making activity or commercial gain

- You may freely distribute the URL identifying the publication in the public portal

If you believe that this document breaches copyright please contact us providing details, and we will remove access to the work immediately and investigate your claim. 


\title{
Efficient Calculation of Born Scattering for Fixed-Offset Ground-Penetrating Radar Surveys
}

\author{
Peter Meincke, Member, IEEE
}

\begin{abstract}
A formulation is presented for efficient calculation of linear electromagnetic scattering by buried penetrable objects, as involved in the analysis of fixed-offset ground-penetrating radar (GPR) systems. The actual radiation patterns of the GPR antennas are incorporated in the scattering calculation by using their plane-wave transmitting and receiving spectra.
\end{abstract}

Index Terms-Born approximation, buried objects, fixed offset, ground-penetrating radar (GPR), microwave imaging, plane-wave transmitting and receiving spectra, scattering.

\section{INTRODUCTION}

$\mathbf{S}$ IGNAL-PROCESSING algorithms based on linear inverse scattering are often used for ground-penetrating radar (GPR) imaging [1]-[8]. To validate and investigate the performance of such algorithms, an efficient calculation of the linear electromagnetic scattering by buried objects—obtained by using the first Born approximation-is essential. However, most GPR systems operate in a fixed-offset mode in which the transmitter and receiver move together, so that a new scattering problem must be solved for each new receiver location. Hence, a straightforward implementation of the Born scattering formulation is too time consuming for analysis of realistic fixed-offset GPR surveys.

In this letter, we derive an efficient formulation for calculating Born scattering by buried dielectric objects in a fixedoffset GPR configuration. Since the formulation is based on plane-wave expansions, it can be efficiently calculated using fast Fourier transforms (FFTs). Also, we include the actual radiation patterns of the GPR antennas by using their planewave transmitting and receiving spectra [9]. The fact that the presence of the air-soil interface impacts the radiation patterns of the antennas is accounted for by the transmitting and receiving spectra.

Throughout this letter, the time factor $\exp (-i \omega t)$ is assumed and suppressed.

\section{FIXED-OFFset GPR CONFIGURATION}

The GPR configuration involving the planar air-soil interface is shown in Fig. 1. A Cartesian $x y z$ coordinate system is introduced so that the $x y$ plane coincides with the interface, and so that $z \geq 0$ is air, and $z<0$ is soil. The air has permittivity $\epsilon_{0}$, permeability $\mu_{0}$, and wavenumber $k_{0}=\omega \sqrt{\mu_{0} \epsilon_{0}}$. The

Manuscript received May 18, 2006; revised September 1, 2006.

The author is with the Ørsted DTU, Electromagnetic Systems, Technical University of Denmark, 2800 Lyngby, Denmark (e-mail: pme@oersted.dtu.dk).

Digital Object Identifier 10.1109/LGRS.2006.886423
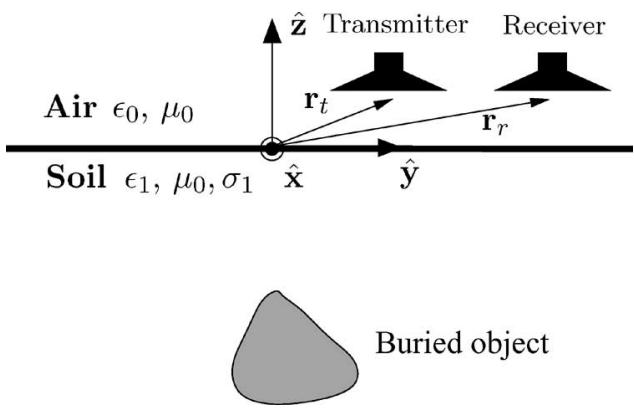

Fig. 1. Fixed-offset GPR configuration in which $\mathbf{r}_{t}=\mathbf{r}_{r}+\mathbf{r}_{\Delta}$ with $\mathbf{r}_{\Delta}$ is fixed.

soil has permittivity $\epsilon_{1}$, conductivity $\sigma_{1}$, permeability $\mu_{0}$, and wavenumber $k_{1}=\sqrt{\omega^{2} \mu_{0} \epsilon_{1}+i \omega \mu_{0} \sigma_{1}}$. The position of the receiving antenna is $\mathbf{r}_{r}=x_{r} \hat{\mathbf{x}}+y_{r} \hat{\mathbf{y}}+z_{r} \hat{\mathbf{z}}$ with $z_{r} \geq 0$, and the position of the transmitting antenna is $\mathbf{r}_{t}=\mathbf{r}_{r}+\mathbf{r}_{\Delta}$ with $\mathbf{r}_{\Delta}=x_{\Delta} \hat{\mathbf{x}}+y_{\Delta} \hat{\mathbf{y}}$ being fixed. Below the air-soil interface, penetrable objects of volume $V_{s}$ are buried. The transmitting antenna is attached to a coaxial cable. In a given reference plane in the cable, the voltage between the inner and outer conductor of the field propagating toward the antenna in the cable is $V_{t}$. The background field $\mathbf{E}^{\mathrm{b}}$, which is defined as the field that would exist in the region $z<0$ in the absence of the objects, is then expressed in terms of the plane-wave transmitting spectrum $\mathbf{T}$ of the GPR antenna as [9]

$$
\begin{array}{r}
\mathbf{E}^{\mathrm{b}}(\mathbf{r})=\frac{V_{t}}{(2 \pi)^{2}} \int_{-\infty}^{\infty} \mathbf{T}\left(k_{x}, k_{y}\right) \exp \left(i \left[k_{x}\left(x-x_{r}-x_{\Delta}\right)\right.\right. \\
\left.\left.+k_{y}\left(y-y_{r}-y_{\Delta}\right)-\gamma_{1} z\right]\right) d k_{x} d k_{y}
\end{array}
$$

where $\mathbf{r}=x \hat{\mathbf{x}}+y \hat{\mathbf{y}}+z \hat{\mathbf{z}}$ with $z<0$ being the position vector, and $\gamma_{1}=\gamma_{1}\left(k_{x}, k_{y}\right)=\sqrt{k_{1}^{2}-k_{x}^{2}-k_{y}^{2}}$, with $\operatorname{Im} \gamma_{1} \geq 0$ being the $z$ component of the propagation vector in the soil. As described in [9], the plane-wave transmitting spectrum $\mathbf{T}$ of the GPR antenna can be determined either from the current density on the antenna or by measurements using a buried probe [10]. Here, we use the former approach and assume that $\mathbf{J}(\mathbf{r})$ is the electric current density on the antenna when it is located at the position $z_{t} \hat{\mathbf{z}}$. The plane-wave transmitting spectrum is then [9]

$$
\mathbf{T}\left(k_{x}, k_{y}\right)=-\frac{\omega \mu_{0}}{2 V_{t}} \overline{\mathbf{F}}\left(k_{x}, k_{y}\right) \cdot \tilde{\mathbf{J}}\left(\mathbf{k}_{0}^{-}\right)
$$

where $\quad \mathbf{k}_{0}^{-}=k_{x} \hat{\mathbf{x}}+k_{y} \hat{\mathbf{y}}-\gamma_{0} \hat{\mathbf{z}}, \quad \gamma_{0}=\gamma_{0}\left(k_{x}, k_{y}\right)=$ $\sqrt{k_{0}^{2}-k_{x}^{2}-k_{y}^{2}}$, and $\operatorname{Im} \gamma_{0} \geq 0$. Moreover, $\tilde{\mathbf{J}}$ is the 
three-dimensional (3-D) spatial Fourier transform of the electric current density given by

$$
\tilde{\mathbf{J}}(\mathbf{k})=\int_{z \geq 0} \mathbf{J}(\mathbf{r}) \exp (-i \mathbf{k} \cdot \mathbf{r}) d^{3} \mathbf{r}
$$

where $\mathbf{k}=k_{x} \hat{\mathbf{x}}+k_{y} \hat{\mathbf{y}}+k_{z} \hat{\mathbf{z}}$. The dyadic $\overline{\mathbf{F}}$ in (2) is related to the dyadic Green's function of a two-layer medium in the spectral domain and is given by

$$
\begin{aligned}
\overline{\mathbf{F}}\left(k_{x}, k_{y}\right)= & \frac{2}{\left(\gamma_{0}+\gamma_{1}\right)\left(k_{x}^{2}+k_{y}^{2}+\gamma_{0} \gamma_{1}\right)} \\
& \cdot\left[\hat{\mathbf{x}}\left(\left(k_{y}^{2}+\gamma_{0} \gamma_{1}\right) \hat{\mathbf{x}}-k_{x} k_{y} \hat{\mathbf{y}}+k_{x} \gamma_{1} \hat{\mathbf{z}}\right)\right. \\
& +\hat{\mathbf{y}}\left(-k_{x} k_{y} \hat{\mathbf{x}}+\left(k_{x}^{2}+\gamma_{0} \gamma_{1}\right) \hat{\mathbf{y}}+k_{y} \gamma_{1} \hat{\mathbf{z}}\right) \\
& \left.+\hat{\mathbf{z}}\left(k_{x} \gamma_{0} \hat{\mathbf{x}}+k_{y} \gamma_{0} \hat{\mathbf{y}}+\left(k_{x}^{2}+k_{y}^{2}\right) \hat{\mathbf{z}}\right)\right] .
\end{aligned}
$$

If the antenna is described by a magnetic current density $\mathbf{M}(\mathbf{r})$, when it is located at the position $z_{t} \hat{\mathbf{z}}$, the plane-wave transmitting spectrum is given by

$$
\mathbf{T}\left(k_{x}, k_{y}\right)=-\frac{1}{2 V_{t}}\left[\mathbf{k}_{1}^{-} \times \overline{\mathbf{F}}\left(k_{x}, k_{y}\right)\right] \cdot \tilde{\mathbf{M}}\left(\mathbf{k}_{0}^{-}\right)
$$

where $\mathbf{k}_{1}^{-}=k_{x} \hat{\mathbf{x}}+k_{y} \hat{\mathbf{y}}-\gamma_{1} \hat{\mathbf{z}}$

\section{BORn SCATTERING BY BURIED OBJECTS}

The receiving antenna of the GPR is connected to a matched receiver through a coaxial cable with characteristic admittance $Y_{0}$. In a given reference plane in the cable, the voltage $V$ between the inner and outer conductors of the field propagating away from the antenna in the cable is [9]

$$
\begin{aligned}
V\left(\mathbf{r}_{r}\right)=\frac{1}{(2 \pi)^{2}} \int_{-\infty}^{\infty} \int_{-\infty} \mathbf{R}\left(k_{x}, k_{y}\right) \cdot \mathbf{S}_{1}^{+}\left(k_{x}, k_{y}\right) \\
\left.\cdot \exp \left(i\left[k_{x} x_{r}+k_{y} y_{r}\right)\right]\right) d k_{x} d k_{y}
\end{aligned}
$$

where $\mathbf{R}\left(k_{x}, k_{y}\right)$ is the plane-wave receiving spectrum of the GPR antenna, which will be discussed below after (11), and $\mathbf{S}_{1}^{+}\left(k_{x}, k_{y}\right)$ is the plane-wave spectrum of the upwardpropagating field $\mathbf{E}_{1}^{+}$in the soil. The plane-wave spectrum $\mathbf{S}_{1}^{+}\left(k_{x}, k_{y}\right)$ is determined by first considering the field $\mathbf{E}_{1}^{+}$. Under the first Born approximation, this field is identical to the scattered field $\mathbf{E}^{s}$ in the soil above the buried object, which is determined by [11, p. 485]

$$
\mathbf{E}^{s}(\mathbf{r})=i \omega \mu_{0} \int_{V_{s}} \overline{\mathbf{G}}\left(\mathbf{r}, \mathbf{r}^{\prime}\right) \cdot \mathbf{E}^{\mathrm{b}}\left(\mathbf{r}^{\prime}\right) O\left(\mathbf{r}^{\prime}\right) d^{3} \mathbf{r}^{\prime}
$$

where $z<0, z>z^{\prime}, \mathbf{r}^{\prime}=x^{\prime} \hat{\mathbf{x}}+y^{\prime} \hat{\mathbf{y}}+z^{\prime} \hat{\mathbf{z}}$, and $O(\mathbf{r})$ is the object function describing the contrast in the electromagnetic properties from those of the background

$$
O(\mathbf{r})=\sigma(\mathbf{r})-\sigma_{1}-i \omega\left(\epsilon(\mathbf{r})-\epsilon_{1}\right) .
$$

Herein, $\epsilon(\mathbf{r})$ and $\sigma(\mathbf{r})$ are the permittivity and conductivity distributions, respectively. Furthermore, $\overline{\mathbf{G}}\left(\mathbf{r}, \mathbf{r}^{\prime}\right)$ in (7) is the dyadic Green's function for a homogeneous medium with wavenumber $k_{1}$ [11, p. 381]

$$
\overline{\mathbf{G}}\left(\mathbf{r}, \mathbf{r}^{\prime}\right)=\left(\overline{\mathbf{I}}+\frac{\nabla \nabla}{k_{1}^{2}}\right) g\left(\mathbf{r}, \mathbf{r}^{\prime}\right) \quad z>z^{\prime}
$$

where $g\left(\mathbf{r}, \mathbf{r}^{\prime}\right)$ is the usual scalar 3-D Green's function for a homogeneous medium

$$
g\left(\mathbf{r}, \mathbf{r}^{\prime}\right)=\frac{\exp \left(i k_{1}\left|\mathbf{r}-\mathbf{r}^{\prime}\right|\right)}{4 \pi\left|\mathbf{r}-\mathbf{r}^{\prime}\right|} .
$$

The dyadic Green's function in (9) does not involve contributions due to the reflection in the interface since these contributions are neglected when applying the first Born approximation. The second step to obtain $\mathbf{S}_{1}^{+}\left(k_{x}, k_{y}\right)$ is to insert the plane-wave expansion of the dyadic Green's function (9) [11, p. 384] into (7) for $\mathbf{E}^{s}$, yielding

$$
\mathbf{S}_{1}^{+}\left(k_{x}, k_{y}\right)=\frac{-\omega \mu_{0}}{2 \gamma_{1}}\left(\overline{\mathbf{I}}-\frac{\mathbf{k}_{1} \mathbf{k}_{1}}{k_{1}^{2}}\right) \cdot\left[\widetilde{O \mathbf{E}^{\mathrm{b}}}\right]\left(\mathbf{k}_{1}\right) .
$$

Herein, $\mathbf{k}_{1}=k_{x} \hat{\mathbf{x}}+k_{y} \hat{\mathbf{y}}+\gamma_{1} \hat{\mathbf{z}}$, and $\left.\widetilde{O \mathbf{E}^{\mathrm{b}}}\right]$ is the 3-D spatial Fourier transform of the product $O(\mathbf{r}) \mathbf{E}^{\mathrm{b}}(\mathbf{r})$.

The plane-wave receiving spectrum $\mathbf{R}\left(k_{x}, k_{y}\right)$ in (6) satisfies that $\mathbf{R}\left(k_{x}, k_{y}\right) \cdot \mathbf{k}_{1}=0$ [9]. For a reciprocal GPR antenna, the receiving spectrum is related to the transmitting spectrum $\mathbf{T}_{r}\left(k_{x}, k_{y}\right)$ of the receiving GPR antenna, defined as in (1) with $V_{t}$ replaced by $V_{\mathrm{tr}}$, as [9]

$$
\mathbf{R}\left(k_{x}, k_{y}\right)=\frac{\gamma_{1}}{\omega \mu_{0} Y_{0}} \mathbf{T}_{r}\left(-k_{x},-k_{y}\right) .
$$

The final expression for the output voltage $V$ is obtained by inserting the plane-wave spectrum (11) into (6)

$$
\begin{aligned}
& \left.V\left(\mathbf{r}_{r}\right)=\frac{-\omega \mu_{0}}{8 \pi^{2}} \int_{-\infty}^{\infty} \int_{-\infty} \frac{1}{\gamma_{1}} \mathbf{R}\left(k_{x}, k_{y}\right) \cdot \widetilde{O \mathbf{E}^{\mathrm{b}}}\right]\left(\mathbf{k}_{1}\right) \\
& \cdot \exp \left(i\left[k_{x} x_{r}+k_{y} y_{r}\right]\right) d k_{x} d k_{y}
\end{aligned}
$$

where the fact that $\mathbf{R}\left(k_{x}, k_{y}\right) \cdot \mathbf{k}_{1}=0$ has been applied. Note that the background field $\mathbf{E}^{\mathrm{b}}$ changes when the radar is moved, and therefore, $\mathbf{E}^{\mathrm{b}}$ needs to be recalculated for each new location of the receiving antenna. Hence, with $N_{z}$ denoting the number of grid points used to discretize the object function along the $z$ axis and $T_{0}$ being the average calculation time of one operation in the two-dimensional (2-D) FFT, the asymptotic calculation time for the straightforward evaluation of (13) for $N_{x} \times N_{y}$ values of $x_{r}, y_{r}$ on an $N_{x} \times N_{y}$ rectangular grid is $6 N_{z} T_{0} N_{x}^{2} N_{y}^{2} \log _{2}\left(N_{x} N_{y}\right)$ as $N_{x}, N_{y} \rightarrow \infty$. Consequently, this calculation scheme is very inefficient for fixed-offset configurations. In the following section, an efficient method is derived to deal with the considered fixed-offset case.

\section{DeRIVING THE EFFICIENT Formulation}

To derive the efficient method, insert first the expression (1) for the background field $\mathbf{E}^{\mathrm{b}}$, with $k_{x}, k_{y}$ replaced by $k_{x}^{\prime}, k_{y}^{\prime}$, into the relation (13) for the output voltage. Second, inspired by 
the procedure in [12], interchange the integrations over $k_{x}, k_{y}$ and $k_{x}^{\prime}, k_{y}^{\prime}$ and carry out the substitutions $k_{x}^{\prime \prime}=k_{x}-k_{x}^{\prime}$ and $k_{y}^{\prime \prime}=k_{y}-k_{y}^{\prime}$. The result is

$$
V\left(\mathbf{r}_{r}\right)=\int_{-\infty}^{\infty} \int_{-\infty} F\left(k_{x}^{\prime \prime}, k_{y}^{\prime \prime}\right) \exp \left(i\left[k_{x}^{\prime \prime} x_{r}+k_{y}^{\prime \prime} y_{r}\right]\right) d k_{x}^{\prime \prime} d k_{y}^{\prime \prime}
$$

with

$$
\begin{aligned}
F\left(k_{x}^{\prime \prime}, k_{y}^{\prime \prime}\right)= & \int_{z^{\prime} \in V_{s}} H\left(k_{x}^{\prime \prime}, k_{y}^{\prime \prime}, z^{\prime}\right) S\left(k_{x}^{\prime \prime}, k_{y}^{\prime \prime}, z^{\prime}\right) d z^{\prime} \\
H\left(k_{x}^{\prime \prime}, k_{y}^{\prime \prime}, z^{\prime}\right)= & \iint_{\left(x^{\prime}, y^{\prime}\right) \in V_{s}} O\left(\mathbf{r}^{\prime}\right) \\
& \cdot \exp \left(-i\left[k_{x}^{\prime \prime} x^{\prime}+k_{y}^{\prime \prime} y^{\prime}\right]\right) d x^{\prime} d y^{\prime} \\
S\left(k_{x}^{\prime \prime}, k_{y}^{\prime \prime}, z^{\prime}\right)= & \iint_{-\infty}^{\infty} \mathbf{C}_{1}\left(k_{x}, k_{y}, z^{\prime}\right) \\
& \cdot \mathbf{C}_{2}\left(k_{x}^{\prime \prime}-k_{x}, k_{y}^{\prime \prime}-k_{y}, z^{\prime}\right) d k_{x} d k_{y} \\
\mathbf{C}_{1}\left(k_{x}, k_{y}, z^{\prime}\right)= & \frac{-\omega \mu_{0} V_{t}}{32 \pi^{4} \gamma_{1}} \mathbf{R}\left(k_{x}, k_{y}\right) \exp \left(-i \gamma_{1} z^{\prime}\right)
\end{aligned}
$$

and

$$
\mathbf{C}_{2}\left(k_{x}, k_{y}, z^{\prime}\right)=\exp \left(i\left[k_{x} x_{\Delta}+k_{y} y_{\Delta}-\gamma_{1} z^{\prime}\right]\right) \mathbf{T}\left(-k_{x},-k_{y}\right) .
$$

The integrations over $k_{x}^{\prime \prime}, k_{y}^{\prime \prime}$ in (14) for $V$ can be determined for all needed receiver locations using one single 2-D FFT. The relation for $H$ in (16) can be calculated analytically for simple object functions, or otherwise by 2-D FFTs for each $z^{\prime}$ and for all needed values of $k_{x}^{\prime \prime}, k_{y}^{\prime \prime}$. The integrations over $k_{x}, k_{y}$ of the dot product $\mathbf{C}_{1}\left(k_{x}, k_{y}, z^{\prime}\right) \cdot \mathbf{C}_{2}\left(k_{x}^{\prime \prime}-k_{x}, k_{y}^{\prime \prime}-k_{y}, z^{\prime}\right)$ in (17) for $S$ are in convolutional form, and therefore, for a fixed $z^{\prime}, S\left(k_{x}^{\prime \prime}, k_{y}^{\prime \prime}, z^{\prime}\right)$ can be calculated for all needed values of $k_{x}^{\prime \prime}$ and $k_{y}^{\prime \prime}$ using 2-D FFTs as follows:

$$
\begin{aligned}
S\left(k_{x}^{\prime \prime}, k_{y}^{\prime \prime}, z^{\prime}\right) & =(2 \pi)^{2} \int_{-\infty}^{\infty} \tilde{\mathbf{C}}_{1}\left(x, y, z^{\prime}\right) \\
& \cdot \tilde{\mathbf{C}}_{2}\left(x, y, z^{\prime}\right) \exp \left(-i\left[k_{x}^{\prime \prime} x+k_{y}^{\prime \prime} y\right]\right) d x d y
\end{aligned}
$$

where

$$
\begin{aligned}
\tilde{\mathbf{C}}_{1}\left(x, y, z^{\prime}\right)=\frac{1}{(2 \pi)^{2}} \int_{-\infty}^{\infty} \int_{-\infty} \mathbf{C}_{1}\left(k_{x}, k_{y}, z^{\prime}\right) \\
\cdot \exp \left(i\left[k_{x} x+k_{y} y\right]\right) d k_{x} d k_{y}
\end{aligned}
$$

and similarly for $\tilde{\mathbf{C}}_{2}$. The functions $\mathbf{C}_{1}$ and $\mathbf{C}_{2}$ in (18) and (19) contain the factor $\exp \left(-i \gamma_{1} z^{\prime}\right)$, which is exponentially decaying for $k_{x}^{2}+k_{y}^{2}>\operatorname{Re} k_{1}^{2}$. Hence, $\mathbf{C}_{1}$ and $\mathbf{C}_{2}$ are spatially bandlimited with the bandwidth $k_{\max }$ determined by requiring that $\exp \left(-\sqrt{k_{\max }^{2}-k_{1}^{2}}\left|z^{\prime}\right|\right)$, with $k_{\max }>\operatorname{Re} k_{1}$, is sufficiently small. The integrations over $k_{x}, k_{y}$ in (17) extend over the ranges $-k_{\max }<k_{x}<k_{\max }$ and $-k_{\max }<k_{y}<$ $k_{\max }$. Equations (15) and (17) then show that the functions
$F\left(k_{x}^{\prime \prime}, k_{y}^{\prime \prime}\right)$ and $S\left(k_{x}^{\prime \prime}, k_{y}^{\prime \prime}, z^{\prime}\right)$ are bandlimited with the bandwidth $2 k_{\text {max }}$. Hence, the grid, on which the output voltage (14) is calculated using FFTs, has the spacing $\Delta x_{r}=\Delta y_{r}=\pi /\left(2 k_{\max }\right)$. Assuming the worst case scenario, in which no closed-form expression exists for $H$ in (16) and 2-D FFTs therefore are needed for its evaluation, the asymptotic calculation time for (14) equals $\left(1+8 N_{z}\right) T_{0} N_{x} N_{y} \log _{2}\left(N_{x} N_{y}\right)$ as $N_{x}, N_{y} \rightarrow$ $\infty$, where $N_{x}, N_{y}, N_{z}$, and $T_{0}$ are defined in the previous section. Hence, the efficient formulation is $6 N_{x} N_{y} N_{z} /$ $\left(1+8 N_{z}\right) \approx 0.75 N_{x} N_{y}$ times faster than the straightforward approach in (13).

\section{A. 2.5-Dimensional Case}

In GPR analysis, it is often of interest to calculate scattering by buried objects that are invariant in one direction. This is, for instance, the case for pipes. In the 2.5-dimensional (2.5-D) case considered in this section, it is assumed that the buried object is invariant in the $\hat{\mathbf{x}}$ direction, and that the antennas are $\hat{\mathbf{x}}$-directed Hertzian dipoles. The $\hat{\mathbf{x}}$-polarized antennas are considered for simplicity, but without loss of generality; other antenna polarization can easily be analyzed as well. The 2.5-D versions of (14)-(19) become

$$
\begin{aligned}
V\left(y_{r}\right) & =\int_{-\infty}^{\infty} F\left(k_{y}^{\prime \prime}\right) \exp \left(i k_{y}^{\prime \prime} y_{r}\right) d k_{y}^{\prime \prime} \\
F\left(k_{y}^{\prime \prime}\right)= & 2 \pi \int_{z^{\prime} \in V_{s}} H\left(k_{y}^{\prime \prime}, z^{\prime}\right) S\left(k_{y}^{\prime \prime}, z^{\prime}\right) d z^{\prime} \\
H\left(k_{y}^{\prime \prime}, z^{\prime}\right)= & \int_{y^{\prime} \in V_{s}} O\left(y^{\prime}, z^{\prime}\right) \exp \left(-i k_{y}^{\prime \prime} y^{\prime}\right) d y^{\prime} \\
S\left(k_{y}^{\prime \prime}, z^{\prime}\right)= & \int_{-\infty}^{\infty} \mathbf{C}_{1}\left(k_{x}, k_{y}, z^{\prime}\right) \\
& \cdot \mathbf{C}_{2}\left(-k_{x}, k_{y}^{\prime \prime}-k_{y}, z^{\prime}\right) d k_{x} d k_{y} \\
& (2 \pi)^{2} \int_{-\infty}^{\infty} \tilde{\mathbf{C}}_{1}\left(x, y, z^{\prime}\right) \\
& \cdot \tilde{\mathbf{C}}_{2}\left(x, y, z^{\prime}\right) \exp \left(-i k_{y}^{\prime \prime} y\right) d x d y
\end{aligned}
$$

where $\tilde{\mathbf{C}}_{1}$ and $\tilde{\mathbf{C}}_{2}$ are defined according to (21) and hence

$$
\begin{aligned}
\mathbf{C}_{1}\left(k_{x}, k_{y}, z\right)= & \omega \mu_{0} V_{t} \exp \left(i\left[\gamma_{0} z_{r}-\gamma_{1} z^{\prime}\right]\right) \\
& \cdot\left(32 \pi^{4} Y_{0} V_{\mathrm{tr}}\left(\gamma_{0}+\gamma_{1}\right)\left(k_{x}^{2}+k_{y}^{2}+\gamma_{0} \gamma_{1}\right)\right)^{-1} \\
& \cdot\left(\hat{\mathbf{x}}\left(k_{y}^{2}+\gamma_{0} \gamma_{1}\right)-\hat{\mathbf{y}} k_{x} k_{y}-\hat{\mathbf{z}} k_{x} \gamma_{0}\right) \\
\mathbf{C}_{2}\left(k_{x}, k_{y}, z^{\prime}\right)= & -\omega \mu_{0}\left(V_{t}\left(\gamma_{0}+\gamma_{1}\right)\right. \\
& \left.\cdot\left(k_{x}^{2}+k_{y}^{2}+\gamma_{0} \gamma_{1}\right)\right)^{-1} \\
& \cdot \exp \left(i\left[k_{x} x_{\Delta}+k_{y} y_{\Delta}+\gamma_{0} z_{r}-\gamma_{1} z^{\prime}\right]\right) \\
& \cdot\left(\hat{\mathbf{x}}\left(k_{y}^{2}+\gamma_{0} \gamma_{1}\right)-\hat{\mathbf{y}} k_{x} k_{y}-\hat{\mathbf{z}} k_{x} \gamma_{0}\right) .
\end{aligned}
$$




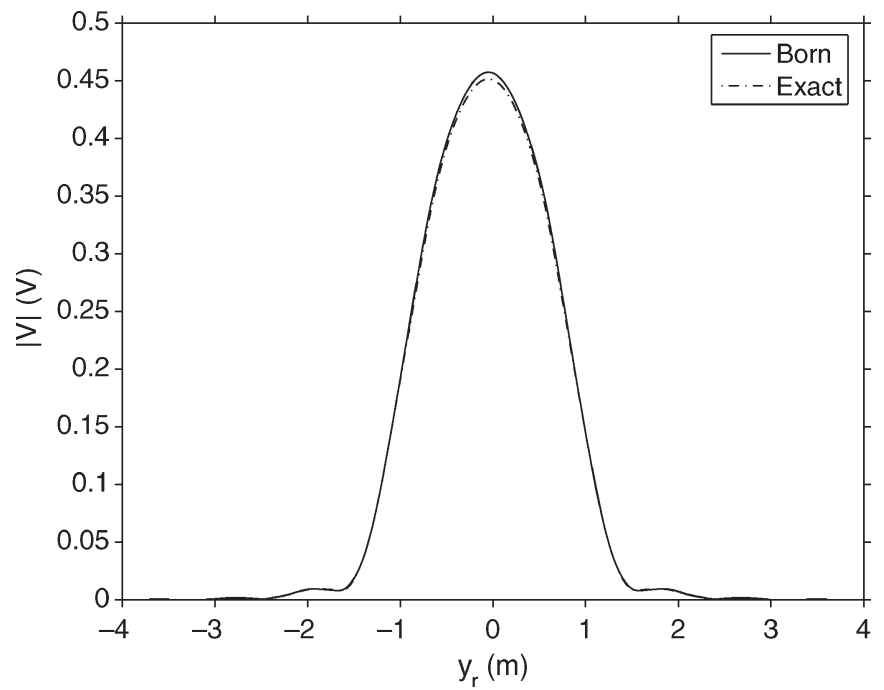

Fig. 2. Magnitude of the output voltage calculated by the Born approximation-based method in this letter and the exact method in [12].

Assume now that the object is a homogeneous cylinder with permittivity $\epsilon_{s}$, conductivity $\sigma_{s}$, axis at the depth $z=z_{0}$, and with rectangular cross section of dimension $2 a \times 2 b$. In this case, $H\left(k_{y}^{\prime \prime}, z^{\prime}\right)$ in (24) becomes

$$
\begin{aligned}
H\left(k_{y}^{\prime \prime}, z^{\prime}\right) & =\int_{-b}^{\mathrm{b}} O\left(z^{\prime}\right) \exp \left(-i k_{y}^{\prime \prime} y^{\prime}\right) d y^{\prime} \\
& =O\left(z^{\prime}\right) 2 b \operatorname{sinc}\left(k_{y}^{\prime \prime} b\right)
\end{aligned}
$$

where

$$
O\left(z^{\prime}\right)= \begin{cases}\sigma_{s}-\sigma_{1}-i \omega\left(\epsilon_{s}-\epsilon_{1}\right), & \left|z^{\prime}-z_{0}\right|<a \\ 0, & \text { otherwise }\end{cases}
$$

Similarly, in case the homogeneous cylinder has circular cross section with radius $a, H\left(k_{y}^{\prime \prime}, z^{\prime}\right)$ becomes

$$
\begin{aligned}
H\left(k_{y}^{\prime \prime}, z^{\prime}\right)=2 O\left(z^{\prime}\right) \sqrt{a^{2}-\left(z^{\prime}-z_{0}\right)^{2}} \\
\cdot \operatorname{sinc}\left(k_{y}^{\prime \prime} \sqrt{a^{2}-\left(z^{\prime}-z_{0}\right)^{2}}\right) .
\end{aligned}
$$

The asymptotic calculation time of the 2.5-D efficient formulation in (22) is $13 T_{0} N_{z} N_{y}^{2} \log _{2} N_{y}$ as $N_{y} \rightarrow \infty$, whereas that of the 2.5-D version of the straightforward procedure in (13) is $3 N_{z} N_{y}^{3} \log _{2} N_{y}$. Hence, in the 2.5-D case, the efficient formulation is $3 N_{y} / 13$ times faster than the straightforward approach.

\section{NUMERICAL INVESTIGATION}

To show the correctness of the efficient formulation derived in Section IV, a 2.5-D case involving scattering by a circular cylinder is considered. The radius of the cylinder is $10 \mathrm{~cm}$, its permittivity and conductivity are $\epsilon_{s}=$ $8.03 \epsilon_{0}$ and $\sigma_{s}=0.01 \mathrm{~S} / \mathrm{m}$, respectively, and it is buried

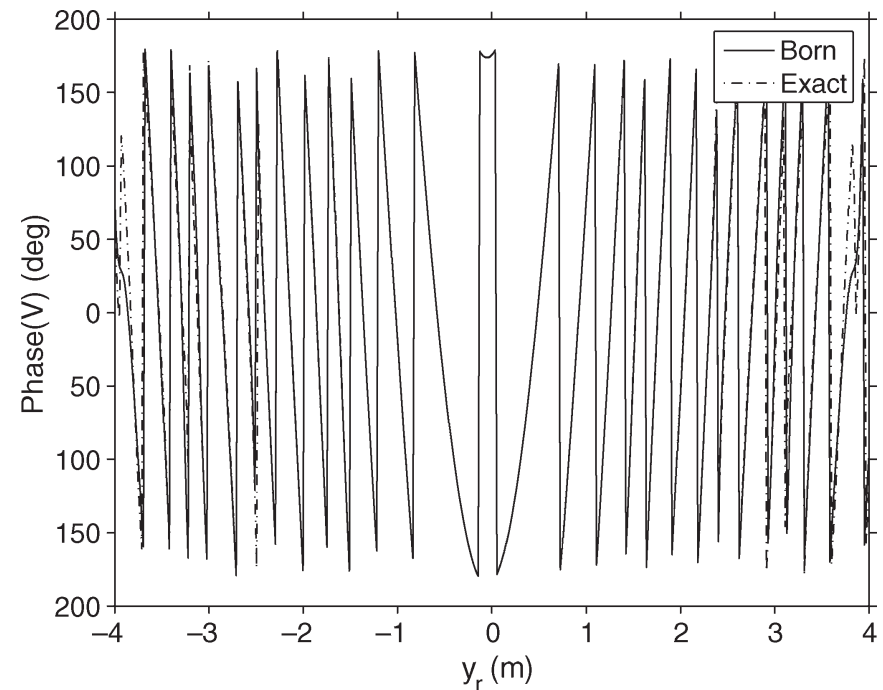

Fig. 3. Phase of the output voltage calculated by the Born approximationbased method in this letter and the exact method in [12].

at $z_{0}=-1 \mathrm{~m}$ in soil with $\epsilon_{1}=8 \epsilon_{0}$ and $\sigma_{1}=0.01 \mathrm{~S} / \mathrm{m}$. The antennas of the GPR are $\hat{\mathbf{x}}$-directed Hertzian dipoles with an offset of $y_{\Delta}=10 \mathrm{~cm}$ and height $5 \mathrm{~cm}$ above the soil, and the radar uses the frequency $241 \mathrm{MHz}$. Equations (22), (23), (25)-(27), and (30) with $V_{t}=V_{\mathrm{tr}}=1 \mathrm{~V}$ and $Y_{0}=20 \mathrm{mS}$ are used to calculate the output voltage $V$ of the receiving Hertzian dipole for 660 values of $y_{r}$ equally spaced between -6 and $6 \mathrm{~m}$.

Figs. 2 and 3 show the magnitude and phase, respectively, of the calculated output voltage compared with the result obtained by using the method described in [12]. The method of [12] is based on an exact eigenfunction expansion of the field scattered by the cylinder. The close agreement between the two results underlines the correctness of the proposed efficient procedure for fixed-offset configurations. The minor discrepancy observed for the magnitude around $y_{r}=0$ is due to the limited accuracy of the first-order Born approximation. For the considered configuration, in which $N_{y}=660$ observation points are considered, the efficient method derived in this letter is approximately $3 N_{y} / 13 \approx 152$ times faster than the straightforward approach.

\section{SUMMARY}

An efficient FFT-based formulation is derived for the calculation of linear scattering by buried objects, as involved in the simulation of fixed-offset GPR surveys. The formulation is particularly useful for validation of linear inverse scattering schemes for fixed-offset GPR configurations. For the 3-D case, in which the fixed-offset GPR is placed on a rectangular grid containing $N_{x} \times N_{y}$ points, the efficient formulation is approximately $0.75 N_{x} N_{y}$ times faster than a straightforward evaluation. Similarly, for the 2.5-D case involving $N_{y}$ observation points, the efficient formulation is $3 N_{y} / 13$ times faster. Ongoing research involves the derivation of a similar efficient formulation based on the nonlinear extended Born approximation [13]. 


\section{REFERENCES}

[1] J. E. Molyneux and A. Witten, "Diffraction tomographic imaging in a monostatic measurement geometry," IEEE Trans. Geosci. Remote Sens., vol. 31, no. 2, pp. 507-511, Mar. 1993.

[2] A. Witten, J. E. Molyneux, and J. E. Nyquist, "Ground penetrating radar tomography: Algorithms and case studies," IEEE Trans. Geosci. Remote Sens., vol. 32, no. 2, pp. 461-467, Mar. 1994.

[3] R. Deming and A. J. Devaney, "A filtered backpropagation algorithm for GPR," J. Environ. Eng. Geophys., vol. 1, no. 2, pp. 113-123, Jan. 1996.

[4] —, "Diffraction tomography for multi-monostatic ground penetrating radar imaging," Inv. Probl., vol. 13, no. 1, pp. 29-45, Feb. 1997.

[5] T. B. Hansen and P. M. Johansen, "Inversion scheme for ground penetrating radar that takes into account the planar air-soil interface," IEEE Trans. Geosci. Remote Sens., vol. 38, no. 1, pp. 496-506, Jan. 2000.

[6] P. Meincke, "Linear GPR inversion for lossy soil and a planar air-soil interface," IEEE Trans. Geosci. Remote Sens., vol. 39, no. 12, pp. 27132721, Dec. 2001.
[7] S. K. Lehman, "Superresolution planar diffraction tomography through evanescent fields," Int. J. Imaging Syst. Technol., vol. 12, no. 1, pp. 1626, Jan. 2002.

[8] J. van der Kruk, C. P. A. Wapenaar, J. T. Fokkema, and P. M. van den Berg, "Three-dimensional imaging of multicomponent ground-penetrating radar data," Geophysics, vol. 68, no. 4, pp. 1241-1254, Jul./Aug. 2003.

[9] P. Meincke and T. B. Hansen, "Plane-wave characterization of antennas close to a planar interface," IEEE Trans. Geosci. Remote Sens., vol. 42, no. 6, pp. 1222-1232, Jun. 2004.

[10] H.-R. Lenler-Eriksen and P. Meincke, "Measurement of plane-wave spectra of ground penetrating radar antennas," Microw. Opt. Technol. Lett., vol. 44, no. 3, pp. 215-216, Feb. 2005.

[11] W. C. Chew, Waves and Fields in Inhomogeneous Media. Piscataway, NJ: IEEE Press, 1995.

[12] T. B. Hansen and P. Meincke, "Scattering from a buried circular cylinder illuminated by a 3-D source," Radio Sci., vol. 37, no. 2, pp. 41-423, Mar. 2002.

[13] T. M. Habashy, R. W. Groom, and B. R. Spies, "Beyond the Born and Rytov approximations: A nonlinear approach to electromagnetic scattering," J. Geophys. Res., vol. 98, no. B2, pp. 1759-1775, Feb. 1993. 